\title{
Unplugging the Universe: the neglected electromagnetic consequence of decoupling
}

\author{
Declan A. Diver and Luís F. A. Teodoro \\ Department of Physics and Astronomy, University of Glasgow, Glasgow, G12 8QQ, Scotland, UK *
}

(Dated: November 19, 2018)

\begin{abstract}
This letter concentrates on the non-equilibrium evolution of magnetic field structures at the onset of recombination, when the charged particle current densities decay as neutrals are formed.

We consider the effect that a decaying magnetic flux has on the acceleration of particles via the transient induced electric field. Since the residual charged-particle number density is small as a result of decoupling, we shall consider the magnetic and electric fields essentially to be imposed, neglecting the feedback from any minority accelerated population.

We find that the electromagnetic treatment of this phase transition can produce energetic electrons scattered throughout the Universe. Such particles could have a significant effect on cosmic evolution in several ways: (i) their presence could delay the effective end of the recombination era; (ii) they could give rise to plasma concentrations that could enhance early gravitational collapse of matter by opposing cosmic expansion to a greater degree than neutral matter could; (iii) they could continue to be accelerated, and become the seed for reionisation at the later epoch $z \approx 10$.

PACS numbers: 98.80.-k, 98.80.Jk, 91.25.Cw, 94.20.wc, 98.58.Ay, 98.62.En, 98.70.Sa
\end{abstract}

In this article, we consider the effect that a decaying magnetic flux has on the acceleration of particles via the transient induced electric field. We do not address the origin of the magnetic field, but merely assume that it exists (see [1] and the references therein).

The Universe underwent recombination at redshift $z \sim$ 1100 , in which the majority of charged particles (i.e. the current-carriers) disappeared as neutrals were formed. This can be understood in the context of simple cosmological models (e.g. [2, 3, 4]). In general, such models do not attempt to describe in detail the actual nonequilibrium processes themselves, but instead simply accommodate their broad consequences.

However there is always a risk, when presenting a series of (quasi) equilibrium states as an evolutionary sequence, of overlooking some essential aspect of the physics that permits the very transition between states. In this article we develop the consequences of describing the transient physics that occurs at decoupling: current densities that decay must inevitably cause an induced electric field, which must accelerate any remaining ambient charged particles. We shall show that this physics cannot be contained within the fluid plasma models that are conventionally used to describe the transition; this is why such essential physics is missing from the overall understanding of universal evolution.

Electromagnetism versus fluid models. - Electromagnetism tells us that magnetic fields are sustained by displacement or charged particle currents. Prior to recombination the high conductivity of the plasma makes the latter the most plausible source of magnetic curvature [5, 6]. After decoupling only a small fraction of electrons $x_{e} \sim 10^{-5}$ [7] persists, meaning that the continuity of the total current must inevitably involve the displacement current replacing the particle current, and consequently the evolution of the pre-decoupling magnetic structures. The accompanying induced electric field will accelerate any remnant charged particles, providing a minority source of energetic plasma that may well be significant in the onward evolution of the cosmos.

Suppose that a typical magnetic field $\boldsymbol{B}_{0}$ pervades a region of characteristic dimension $L_{0}$ and that the particle current which is sustaining it vanishes. How does the system evolve from this point onwards? Electromagnetism tells us that if the particle currents are interrupted, then the overall system will react with an induced electric field in an attempt to oppose the change. The size of the induced electric field is related to the timescale over which the magnetic flux is changing. The subtlety here is ensuring that cosmological models permit the appropriate timescales in the evolutionary processes. Fully electromagnetic descriptions are entirely appropriate, but those that depend on magnetohydrodynamical (MHD) plasmas may well be unable to accommodate the necessary physics at transition, and as a result, underestimate the significance of the decoupling itself.

In the fully electromagnetic description, the electric and magnetic fields $\boldsymbol{E}, \boldsymbol{B}$ and plasma current density $\boldsymbol{J}$ are governed by

$$
\boldsymbol{\nabla} \times \boldsymbol{E}=-\dot{\boldsymbol{B}}, \quad \nabla \times \boldsymbol{B}=\mu_{0} \boldsymbol{J}+\dot{\boldsymbol{E}} / c^{2},
$$

where denotes $\partial / \partial t$. Assuming that the current density $\boldsymbol{J}$ is negligible as a result of decoupling leads to $\boldsymbol{\nabla} \times \boldsymbol{B}$ being determined by $\dot{\boldsymbol{E}} / c^{2}$. The dimensional analysis of (11) yields a timescale for changes in the field evolution of

$$
\tau_{1}=L_{0} / c .
$$

Hence the magnetic field reconfigures by changes which propagate at light speed.

However, if the plasma is modelled using MHD such rapid communication is not permitted, since the displacement current is omitted from MHD dynamics [8]. Hence instead of the full electromagnetic equations, the fluid 
plasma of resistivity $\eta$ is governed by

$$
\begin{gathered}
\boldsymbol{\nabla} \times \boldsymbol{E}=-\dot{\boldsymbol{B}}, \quad \nabla \times \boldsymbol{B}=\mu_{0} \boldsymbol{J}, \\
\boldsymbol{E}+\boldsymbol{u} \times \boldsymbol{B}=\eta \boldsymbol{J},
\end{gathered}
$$

in which $\boldsymbol{u}$ is the plasma velocity. Note that now we cannot ignore $\boldsymbol{J}$ in favour of the displacement current, since the former is the only way in which $\nabla \times \boldsymbol{B}$ is sustained. It is clear at this stage that the sudden loss of electrical current as neutrals form is beyond the compass of such a framework; the magnetic field can only communicate changes at the resistive or fluid-dynamic timescales. This can be seen from the form of Ohm's Law in Eq. (4). Combining Eq.s (3-4) we obtain

$$
\dot{\boldsymbol{B}}=\boldsymbol{\nabla} \times(\boldsymbol{u} \times \boldsymbol{B})-\bar{\eta} \boldsymbol{\nabla}^{2} \boldsymbol{B}
$$

in which $\bar{\eta}=\eta / \mu_{0}=$ constant. In magnitude terms

$$
B_{0} / \tau: u_{0} B_{0} / L_{0}: \bar{\eta} B_{0} / L_{0}^{2} \quad \text { or } \quad 1: u_{0} \tau / L_{0}: \bar{\eta} \tau / L_{0}^{2}
$$

which leads to a typical resistive time scale $\tau_{2}$ given by

$$
\tau_{2}=L_{0}^{2} / \bar{\eta}
$$

assumed to be the characteristic of resistive MHD; the dynamo timescale

$$
\tau_{3}=L_{0} / u_{0}
$$

is the only possibility for an ideal (perfectly conducting) MHD plasma.

The induced electric field. - The magnitudes of the induced electric fields for the different scenarios are given by the induction law, which relates the induced electromotive force to the rate of change of magnetic flux $\Phi$ :

$$
\oint \boldsymbol{E} \cdot \mathrm{d} \boldsymbol{l}=-\frac{\mathrm{d} \Phi}{\mathrm{d} t}=\frac{\partial}{\partial t} \int \boldsymbol{B} \cdot \mathrm{d} \boldsymbol{A}
$$

in which the closed-loop integral is around the path enclosing the area $A$, in the usual way. We have used partial derivatives in time on the right-hand side because we will consider the field at a point in space, rather than the motional emf. If we assume a circular area of radius $L_{0}$ through which the magnetic flux is linked, then we can identify the typical size of induced electric field by dimensional analysis:

$$
E \sim B L_{0} / \tau_{D}
$$

where $\tau_{D}$ characterises the time over which the magnetic field decays at that location, that is, the timescale for the particle current density $\boldsymbol{J}$ to disappear. If we use the timescales associated with the electromagnetic and MHD models, then we arrive at the following estimates of the induced electric field:

$$
\begin{aligned}
\text { electromagnetism } & E_{1}=c B_{0} \\
\text { resistive MHD } & E_{2}=L_{0} / \tau_{2} B_{0}=\bar{\eta} / L_{0} B_{0} \\
\text { ideal MHD } & E_{3}=u_{0} B_{0}
\end{aligned}
$$

Comparing the magnitudes of the fields by forming $E_{1}$ : $E_{2}: E_{3}$ we have

$$
c B_{0}: \bar{\eta} / L_{0} B_{0}: u_{0} B_{0} \quad \text { or } \quad 1: \bar{\eta} / L_{0} c: u_{0} / c
$$

Since MHD (ideal or resistive) must have $u_{0} \ll c$, it is clear from this that the induced electric field permitted by the fully electromagnetic model is the most significant

The key issue here is that the size of the induced electric field is determined by the rate of change of the magnetic flux. Only the electromagnetic model can accommodate the rapidly varying current density that results from recombination (a quantum, rather than fluid, effect). The fluid plasma models tie the evolution of the magnetic field explicitly and directly to the fluid motion via $\boldsymbol{u}$. This is particularly acute in ideal MHD, where the flux linked by a fluid element can never change, and so the only physical process that allows flux to decay overall is cosmic expansion. There is a subtle point here in ideal MHD: the frozen-in flux condition means that the magnetic flux at a point can change only at the expense of plasma motion; however, a decrease at one point leads to an increase somewhere else as the fluid rarifies and compresses. Hence ideal MHD cannot in fact accommodate recombination, since the latter causes an unavoidable overall loss of magnetic flux when the current density (essentially) vanishes - it is not simply a case of rearrangement.

Moreover, the fluid model is further compromised by the fact that the $\boldsymbol{u}$ that appears is the plasma fluid velocity: the very species that is being eliminated as a result of the recombination. Hence tying the induction field to a vanishing fluid species cannot recover the correct physics at decoupling. This explains why the electric field produced in such models is deemed wholly negligible.

The consequence of electric induction. - We will now explore the consequences of an induced electric field, considering only the electromagnetic case. It is vital to distinguish between timescales, however, in order to avoid confusion.

The timescale for transmission of field changes (including boundary conditions) is the electromagnetic one, given by $\tau_{1}$ in Eq. (2); we will drop the subscript 1 from now on.

There is also the characteristic time for the decay of the current density, $\tau_{D}$, which could be very short, since it is governed by atomic processes: for example, by the rate coefficient for the attachment of free electrons to positive ions. The process is strongly density dependent, and therefore influenced by local conditions.

Finally, we can identify a timescale $\tau_{R}$ over which recombination is completed across the universe. This is taken to be of the order of $100 \mathrm{kyr} \sim 10^{12} \mathrm{~s}$. [4]

Hence at a location, changes in the current density due to neutral formation (evolving over the timescale $\tau_{D}$ ) are communicated throughout that location by electromagnetic signals, which take a characteristic time $\tau$ to reach 
all parts. $\tau_{R}$ is the timescale for all such locations in the universe to complete this process.

A simple example: particle acceleration.- Dimensional analysis of Eq. (9) gives the electric field magnitude in terms of the magnetic field and the characteristic length and time:

$$
E=B L_{0} / \tau_{D}
$$

In order to quantify the size of the induced electric field, we must supply typical values for $B, L_{0}$ and $\tau_{D}$. A typical magnetic field value is $B \sim 10^{-12} \mathrm{~T}[9,10]$. The scale-length is more challenging: this could range from $10^{11} \mathrm{~m}$ (an astronomical unit) to $10^{16} \mathrm{~m}$ (a parsec), with the latter being more realistic in the context of structure boundaries visible in [9, 10].

The characteristic time for the recombination to take place at a point is not the recombination width $\tau_{R}$, but the timescale that arises self-consistently from the rate equation for the formation of neutrals. In general terms, the rate equation governing the conversion of one species into another via collisions with electrons takes the form $\dot{n}_{a}=\alpha n_{b} n_{e}$ where here $n_{a}$ plays the role of neutral number density, $n_{b}$ the positive ion number density, and $n_{e}$ the electron number density. The rate coefficient $\alpha$ for recombination [11, 12, 13] is typically $10^{-16} \mathrm{~m}^{3} \mathrm{~s}^{-1}$, yielding

$$
\tau_{D} \sim(n \alpha)^{-1}
$$

where $n$ is a typical number density at the recombination epoch. Taking $n \sim 10^{8} \mathrm{~m}^{-3}$ yields $\tau_{D} \sim 10^{8} \mathrm{~s}$.

Suppose we have a uniform axial magnetic field $\boldsymbol{B}(\boldsymbol{x})$ within some radius $L_{0}$ which is supported by an edgelocated current structure which vanishes at the time of recombination. (A helpful analogy would be a magnetic solenoid.) The magnetic field is given by $\boldsymbol{B}(\boldsymbol{x})=\hat{\boldsymbol{z}} B_{0}$ and $\nabla \times \boldsymbol{B}=0$ except where the current is carried. When the current density is switched off, the induced electric field is non-zero everywhere (except perhaps within the original current carrying region) as can be seen from Eq. (1). We shall assume that the induced field propagates into the uniform magnetic field region at speed $c$, and our analysis will be concerned with the evolution of the system once the current collapse has been communicated across $L_{0}$. This is a reasonable assumption, since we wish to follow the energetic evolution of an inertial test particle initially at rest when the current collapses. Of course, any candidate for acceleration by such fields will initially be in equilibrium with the CMBR, but this straightforward case will highlight the essential physics.

Let us assume that $\boldsymbol{B}$ retains a predominantly axial character during the acceleration of the test particle; there will of course be evolving magnetic curvature, but in the spirit of keeping the problem simple, we will set this aside. Hence the induced electric field will remain primarily azimuthal. Therefore

$$
\rho^{-1}\left(\partial / \partial_{\rho}\right)\left(\rho E_{\phi}\right) \approx-\dot{B}_{0}(t)
$$

where $(\rho, \phi, z)$ are standard cylindrical coordinates. The right-hand side of Eq. (17) is independent of $\rho$ and so we can integrate immediately to get

$$
E_{\phi}(\rho, t) \approx-\dot{B}_{0}(t) \rho / 2
$$

Given these approximate fields, we can now turn to the trajectory of an electron that is subject to them. It is appropriate to consider the single-particle behaviour here, since we are describing the evolution of a minority population of charged particles that escape elimination from neutral recombination. We know that the ionization fraction drops by 5 orders of magnitude at recombination [7]; it is reasonable then to consider single-particle effects without taking into account any bulk feedback from the motion of such charges. Note that the particle being accelerated here needn't necessarily be an original member of the particle current ensemble that sustained the pre-decoupling magnetic field.

The relativistic Lorentz equation for an electron moving under imposed electric and magnetic fields is

$$
\mathrm{d} \boldsymbol{p} / \mathrm{d} t=-e(\boldsymbol{E}+\boldsymbol{v} \times \boldsymbol{B})
$$

where $\boldsymbol{p}=\gamma m_{e} \boldsymbol{v}$ is the electron 3-momentum, and $\gamma=1 / \sqrt{1-v^{2} / c^{2}}$. The reference (or lab) frame here is the one in which the magnetic field is predominantly axial, and the electric field azimuthal, consistent with our model configuration.

If this particle acquires the drift speed $\boldsymbol{u}_{d}=$ $\boldsymbol{E} \times \boldsymbol{B} / B^{2}$ in the lab frame then the Lorentz force vanishes, and the particle is free, having energy $\mathcal{E}$ in addition to its rest energy given by $\mathcal{E}=(\gamma-1) m_{e} c^{2}$. We can take $\mathcal{E}$ to be the maximum energy gain for a particle initially at rest (or in thermal equilibrium at decoupling) before being subjected to the induced electric field that must accompany the collapsing current density. For $E / B \approx 5 \times 10^{7} \mathrm{~ms}^{-1}$, then $\gamma \approx 1.014$ and so such an electron has gained approximately $1.4 \%$ of its rest energy, or about $7 \mathrm{keV}$, as a direct result of the decoupling process.

This result can be achieved in a different way. Consider a thermal electron at decoupling, moving in a Larmor orbit in our typical magnetic field of $1 \mathrm{pT}$. Using $v_{\perp} \approx$ $3 \times 10^{5} \mathrm{~ms}^{-1}$ and $\omega_{c}=e B_{0} / m_{e} \approx 0.1 \mathrm{~Hz}$, the Larmor radius $R_{L}=v / \omega_{c}$ at decoupling is $R_{L 0} \sim 3 \times 10^{6} \mathrm{~m}$. Since the induced electric field is predominantly azimuthal, the change in azimuthal speed $\delta v$ resulting from the azimuthal acceleration is $\delta v \approx\left(e / m_{e}\right) E \delta t$, leading to an increase in Larmor radius of $\delta L_{R} \approx\left(e / \omega_{c} m_{e}\right) E \delta t$. Taking the outward drift speed of the particle as the rate of change of its Larmor radius, we arrive at $\mathrm{d} R_{L} / \mathrm{d} t \approx E / B$, as before. Substituting for $E$ using Eq. (18), we can solve for the time evolution of the outward drift:

$$
R_{L}(t) \approx R_{L 0}\left(B_{0} / B(t)\right)^{1 / 2}
$$

where $B_{0}$ is the magnetic field just prior to decoupling. If we assume that the current density decays exponentially with characteristic time $\tau_{D}$, then it is clear that 
the Larmor radius of the particle reaches $1 \mathrm{pc}$ after a time $t \approx 40 \tau_{D}$, at which point it is travelling with a radial speed $\mathrm{d} R_{L} / \mathrm{d} t \approx R_{L 0} /\left(2 \tau_{D}\right) \approx c / 6$, as before. Note that this outward drift speed dominates the azimuthal component.

This modelling underlines the fact that the elimination of magnetic energy at decoupling creates an electromagnetic impulse which accelerates ambient charged particles.

Conclusions. - In this letter we model the transient magnetic and electric fields at the time of recombination, distinguishing between three critical timescales: (i) $\tau$, the time taken for fields to accommodate the evolving current density; (ii) $\tau_{D}$, the time for recombination to take place in a localised region; and (iii) $\tau_{R}$ the time window for decoupling to be completed across the universe.

Dimensional analysis shows that only a full electromagnetic model allows $\tau$ to be consistent with the appropriate physics: recombination is a relatively rapid event driven by the relevant rate coefficients and number densities of participating species, and not by a global fluid (MHD) model that cannot incorporate rapid changes in current density, and in the case of ideal MHD, cannot allow a global change in the magnetic flux. Such MHD models inevitably link field evolution to bulk fluid motion, thereby imposing an unacceptabe evolution timescale.

The critical features of our model can be summarised as follows. A minority cohort of charged particles (here taken as electrons), initially in thermal equilibrium with the matter in the Universe, survive the recombination process at the boundary between magnetic domains. The rapidly changing current density caused by the elimination of the majority population of free charged particles (recombination) induces an electric field that accelerates any that are left over. The maximum energy that such particles can acquire in this process occurs when they move at the drift velocity, corresponding to an energy of approximately $1.4 \%$ of their rest mass.

We have identified a process in which a population of energetic particles is produced as a direct result of decoupling. Such particles, although very much a minority fraction of the neutral number density, are clearly not in thermal equilibrium with that majority; this may leave a signature in the CMBR, albeit at very low frequency. Moreover they are sufficiently energetic to cause an element of reionisation of neutral matter in the decoupling era, should the collisional environment be appropriate, and in so doing, perhaps delaying the end of the recombination era.

More significantly we have described a mechanism which can seed the Universe with high-energy charged particles from a single, localised decoupling event. Such particles could in principle be accelerated to even greater energies by further encounters with induced fields at different locations, since it is clear that $\tau_{R} \gg \tau_{D}$. Hence our model may offer a route whereby cosmic ray particles could be created.

Finally, although in a minority, the persistence of such energetic plasma beyond the decoupling era may accelerate the condensation of matter into early structure formation, since long-range Coulomb forces may be sufficient to bias gravitational self-collapse.

A full numerical simulation of the magnetic field evolution, energetic particle production and the observational consequences is currently underway [14] and will be reported later.

DAD and LFAT acknowledge support from PPARC and the Leverhulme Trust respectively. Thanks are also due to Tobia Carozzi for helpful comments.

* Enquiries to d.diver@physics.gla.ac.uk

[1] D Grasso and HR Rubinstein. Magnetic fields in the early universe. Physics Reports-Review Section of Physics Letters, 348(3):165 - 266, 2001.

[2] R. Bean, A. Melchiorri, and J. Silk. Phys. Rev. D, 68(8):083501-+, October 2003.

[3] D. N. Spergel, R. Bean, O. Doré, M. R. Nolta, C. L. Bennett, J. Dunkley, G. Hinshaw, N. Jarosik, E. Komatsu, L. Page, H. V. Peiris, L. Verde, M. Halpern, R. S. Hill, A. Kogut, M. Limon, S. S. Meyer, N. Odegard, G. S. Tucker, J. L. Weiland, E. Wollack, and E. L. Wright. ArXiv Astrophysics e-prints, March 2006.

[4] C. L. Bennett, M. Halpern, G. Hinshaw, N. Jarosik, A. Kogut, M. Limon, S. S. Meyer, L. Page, D. N. Spergel, G. S. Tucker, E. Wollack, E. L. Wright, C. Barnes, M. R. Greason, R. S. Hill, E. Komatsu, M. R. Nolta, N. Odegard, H. V. Peiris, L. Verde, and J. L. Weiland. ApJ Suppl., 148:1-27, September 2003.

[5] J. Ahonen and K. Enqvist. Physics Letters B, 382:40-44, 1996.

[6] G. Baym and H. Heiselberg. Phys. Rev. D, 56(8):52545259, 1997.

[7] T. Padmanabhan. Structure formation in the Universe. Cambridge University Press, Cambridge, U.K., 1st edition, 1993.

[8] L.F.A. Teodoro, D.A. Diver, and M.A. Hendry. A cautionary note on cosmological magnetic fields. astro-ph/0702729 submitted to Phys. Rev. D

[9] K. Ichiki, K. Takahashi, H. Ohno, H. Hanayama, and N. Sugiyama. Science, 311:827-829, February 2006.

[10] J. D. Barrow, P. G. Ferreira, and J. Silk. Physical Review Letters, 78:3610-3613, May 1997.

[11] U. Wenzel, K. Behringer, A. Carlson, J. Gafert, B. Napiontek, and A. Thoma. Nuclear Fusion, 39(7):873-882, 1999.

[12] S. Bhattacharyya, A. Roy, and S. Mitra. Fizika A, 6(4):149-160, 1997.

[13] S. Seager, D. D. Sasselov, and D. Scott. ApJ Suppl., 128:407-430, June 2000.

[14] L.F.A. Teodoro and D.A. Diver. The collapse of the cosmological magnetic field at recombination. (in preparation) 